\title{
Reconstruction and future prediction of the sea surface from radar observations
}

\author{
A. P. Wijayaa ${ }^{a, b, *}$ P. Naaijen ${ }^{c}$, Andonowati ${ }^{b, d}$, E. van Groesen ${ }^{a, b}$ \\ ${ }^{a}$ Applied Mathematics, University of Twente, Netherlands \\ ${ }^{b}$ LabMath-Indonesia, Bandung, Indonesia \\ ${ }^{c}$ Maritime 83 Transport Technology, Technical University Delft, Netherlands \\ ${ }^{d}$ Mathematics, Institut Teknologi Bandung, Indonesia
}

\begin{abstract}
For advanced offshore engineering applications the prediction with available nautical X-band radars of phase-resolved incoming waves is very much desired. At present, such radars are already used to detect averaged characteristics of waves, such as the peak period, significant wave height, wave directions and currents. A deterministic prediction of individual waves in an area near the radar from remotely sensed spatial sea states needs a complete simulation scenario such as will be proposed here and illustrated for synthetic sea states and geometrically shadowed images as synthetic radar images. The slightly adjusted shadowed images are used in a dynamic averaging scenario as assimilation data for the ongoing dynamic simulation that evolves the waves towards the near-radar area where no information from the radar is available.

The dynamic averaging and evolution scenario is rather robust, very efficient and produces qualitatively and quantitatively good results. For study cases of wind waves and multi-modal wind-swell seas, with a radar height of 5 times the significant wave height, the correlation between the simulated and the actual sea is found to be at least $90 \%$; future waves can be predicted up to the physically
\end{abstract}

\footnotetext{
${ }^{*}$ Corresponding author at : LabMath-Indonesia, Jl. Dago Giri no 99, Warung Caringin, Mekarwangi, 40391 Bandung, Indonesia. Tel : +62 222507476

Email address: a.parama@labmath-indonesia.org (A. P. Wijaya)
}

Preprint submitted to Ocean Engineering

May 25, 2015 
maximal time horizon with an averaged correlation of more than $80 \%$.

Keywords: remote sensing, sea surface reconstruction, sea surface prediction, multi-modal sea states, radar image, dynamic averaging.

1

2

\section{Introduction}

Attempts to use remote sensing of the sea surface for prediction of the actual and future surface elevation in the vicinity of floating ships or offshore structures is motivated by various offshore and maritime engineering applications. Positioning of vessels would benefit from knowledge of the near future incoming low and high waves. Helicopter landing and loading / off-loading operations with at least one floating structure involved are examples of operations of which the critical phase (touch down or lift off) is conducted preferably during a time window with low waves. These workable time windows may occur as well in relatively high seas making their prediction very valuable to increase operational time. Knowing the approach of a freak wave, which seems to occur much more frequently than previously thought, can help to control ships in a safer way (Clauss et al., 2014). An attractive option for the remote wave sensor is the nautical X-band radar. Much attention has been given since several decades to its application as a wave sensor. The vast majority of the efforts so far has been based on spectral 3D FFT methods dedicated to retrieve statistical wave parameters such as mean wave period, wave direction, non-phase-resolved directional wave spectra and properties that could be derived from the surface elevation like water depth and surface current speed and direction. Young et al. (1985) used spectral analysis to detect currents, and Ziemer and Rosenthal (1987) proposed the use of a modulation transfer function to derive surface elevation from radar images of the sea surface. Borge et al. (1999) used the signal-to-noise (SNR) ratio in radar images to propose an approximate relation for the significant wave height with two parameters that have to be calibrated. The question how to 
reveal the exact relation between radar images and wave elevation / significant wave height has been subject to many more publications, see e.g. Buckley and Aler (1998) and Gangeskar (2014). We will not address this topic here, but refer to a forthcoming publication of Wijaya and van Groesen (2015) that derives the significant wave height from the shadowed images without any calibration. In this paper it is assumed that the significant wave height is known, either from existing analysis techniques of radar images or by means of a reference observation such as a wave buoy or recorded ship motions.

Some of the rare attempts to retrieve the actual deterministic, i.e. phase resolved, wave surface elevation from radar-like images are reported by Blondel and Naaijen (2012) and Naaijen and Blondel (2012), but the quality was shown to be not optimal. A very different method has been explored by Aragh and Nwogu (2008); they use a 4D Var assimilation method, assimilating (raw) radar data in an evolving simulation. Nevertheless, it seems that in literature no statistically significant evidence has been reported for successful deterministic wave sensing (reconstruction), nor any method to propagate the waves to a blind area or to provide predictions.

To overcome the 'blind' zone around the radar where no elevation information is available, a propagation model is needed to evolve phase resolved reconstructed waves in the visible area into the blind zone and to make future predictions of the waves there, e.g. at the position of the ship carrying the radar antenna.

The main aim of this paper is to present a scenario that integrates the inversion of the observed images with the propagation and prediction. This integration is achieved by a robust dynamic averaging-evolution procedure which will be shown to provide a prediction accuracy that is significantly higher than the accuracy of the observation of a single image itself.

In the following we will restrict to the case that the radar position is fixed; im- 
ages from a radar on a ship moving towards the waves will require some obvious adaptations, and will reduce the prediction horizon. The complete evolution scenario takes into account the specific geometry determined by the radar scanning characteristics. For the common nautical X-band radars one can distinguish the ring-shaped area where information from radar scans is available, and the near radar area where this information is missing. Through the outer boundary of the ring, some $2000 \mathrm{~m}$ away from the radar, waves enter and leave the area; part of the incoming waves evolve towards the near-radar area or interact with waves that determine the elevation there. Hence, updates to catch the incoming waves have to be used repeatedly. The inner boundary of the ring determines the disk, the near-antenna area with a radius of some $500 \mathrm{~m}$; there no useful radar information is available because the backscatter is too high and/or suffers from interaction effects with the ship's hull. A propagation model has to evolve the information from the ring area inwards to the radar position. This description defines the main ingredients of a process that has to be developed into a practical scenario that is sufficiently efficient and accurate, noting that the quality of the simulated elevation in the near-radar area depends on the quality of the simulation in the radar ring. Since radar images give only partial and distorted information about the actual sea surface, mainly because of the shadowing effect, a phase resolved reconstruction of the sea - the inversion problem - is important. As we will show, the use of a sequence of images in a spatially dynamic scenario will predict the present and future sea surface in a reasonable degree of accuracy.

We start to propose two simple reconstruction methods for single images, but fail to reduce the effects of shadowing noticeably; consecutive simulations with the raw and the the reconstructed images will provide an indication of the robustness of the complete scenario. Indeed, the quality of the reconstruction will 
be substantially enhanced by the dynamic averaging and evolution procedure, almost independent of the choice of these initial images. The procedure consists of the averaging of a few successive (reconstructed) images, together with the result of the dynamic simulation, to produce updates that are assimilated in the dynamic simulation. We will use the full ring shaped observation domain surrounding the target location; this makes it possible to reconstruct and predict uni-modal wind waves as well as multi-modal seas with wind waves and swell(s) coming from possibly substantially different directions. Specific attention will be paid to the question how to treat the evolution of multi-modal seas in the proposed scenario.

In this paper we use synthetic data and make some simplifications for ease of presentation, but the scenario to be described can also be applied for more realistic cases. The use of synthetic data makes it possible to quantify the quality of the results which will be difficult to achieve in field situations for which reliable data of the surface elevation both in the ring-shaped observation area and the near-radar area simultaneously are very difficult to obtain. The wind and windswell seas that we synthesize are chosen to be linear to simplify the evolution, but linearity is not essential. From the synthetic seas, we construct synthetic radar images by only taking the geometric effect of shadowing into account as an illustration that the scenario can resolve imperfections of that kind.

The paper is arranged according to the successive steps in the proposed scenario. Section 2 will describe the design of (multi-modal) synthetic seas and of synthetic radar images by applying the shadowing effects. In Section 3 the complete dynamic averaging-evolution scenario (DAES) will be described to determine from the shadowed images the wave elevation inside the observable area and inside the blind area near the radar. Section 4 describes the results for two case studies, one case of wind waves, and the other one for wind-swell seas; 
apart from reconstruction results, the quality of predictions are investigated up to the maximal prediction time. In section 5 the results of the study case are discussed and conclusive remarks will be given in section 6 .

\section{Synthetic data}

After a motivation to restrict the investigations to shadowed seas in the first subsection, we describe the construction of the synthetic surface elevation maps. These will be used in subsection 2.3 to generate the synthetic geometric images that take into account the shadowing effect, and later to quantify the quality of the reconstructed and evolved surface elevations.

\subsection{Simplifications}

When the sea will be scanned by the radar, parts of it will be hidden for the electromagnetic radar waves since they are partly blocked by waves closer to the radar, the geometric shadowing. It should be remarked that investigations of radar data by Plant and Farquharson (2012a) do not support the hypothesis that geometric shadowing plays a significant role at low-grazing-angle; indications are found that shadowing rather occurs as so-called partial shadowing. Besides shadowing, tilt (slope of the sea surface relative to the look-direction of the radar) is considered to be an important modulation mechanism for wave observations by radar, see Borge et al. (2004) and Dankert and Rosenthal (2004). In all these references the so-called hydrodynamic modulation as described by e.g. Alpers et al. (1981) has been ignored. Possible other effects perturbing the observation that are introduced by specific hardware related properties of a radar system should in general be invertible when the exact properties are known, which is why we do not consider that aspect here.

In this paper we will consider as example of imperfections of the observed sea the effect of geometric shadowing. For this relevant effect it will be shown how 
well the proposed averaging-evolution scenario can cope with imperfections with a length scale of the order of one wavelength, virtually independent of the precise cause of the imperfections. Since this geometrical approach is mainly valid as a first order approach of the backscattering mechanism for grazing incidence conditions at far range for marine radar (Borge et al., 2004), electromagnetic diffraction (Plant and Farquharson, 2012b) is not taken into account in this paper. It must be noted that perturbations over larger areas as caused by severe wind bursts may not be recovered accurately by the present methods.

\subsection{Synthetic surface elevations}

To synthesize a sea, we use a linear superposition of $N$ regular wave components each having a distinct frequency and propagation direction. The wave spectrum $S_{\eta}(\omega)$ is defined on an equally spaced discrete set of frequencies $\omega_{n}$ covering the significant energy contributions. In order to assure that the sea is ergodic (Jefferys, 1987), it is required that only a single direction corresponds to each frequency. A propagation direction is assigned to each wave component by randomly drawing from the directional spreading function which is used as a probability density function, as proposed by Goda (2010). The directional spreading function with exponent $s$ around the main direction $\theta_{\text {main }}$ is given by

$$
D(\theta)= \begin{cases}\beta \cos ^{2 s}\left(\theta-\theta_{\text {main }}\right), & \text { for }\left|\theta-\theta_{\text {main }}\right|<\pi / 2 \\ 0, & \text { else }\end{cases}
$$

with normalization $\beta$ such that $\int D(\theta) d \theta=1$.

With $k_{n}$ the length of the wave vectors corresponding to the frequencies $\omega_{n}$, and with $\phi_{n}$ phases that are randomly chosen with uniform distribution in 
$[-\pi, \pi]$, the sea is then given by

$$
\eta(\mathbf{x}, t)=\sum_{n} \sqrt{2 S_{\eta}\left(\omega_{n}\right) d \omega} \cos \left(k_{n}\left(x \cos \left(\theta_{n}\right)+y \sin \left(\theta_{n}\right)\right)-\omega_{n} t+\phi_{n}\right)
$$

Snapshots of the surface elevation at multiples of the radar rotation time $d t$ are given by $\eta(\mathbf{x}, n \cdot d t)$.

\subsection{Geometric images}

With 'Geometric Images' we refer to the synthesized radar observation of the surface elevation for which, as stated above, we will only take the geometric shadowing into account. Shadowing along rays has been described by Borge et al. (2004) and is briefly summarized as follows.

After interpolating the image on a polar grid, with the radar at the origin $\mathbf{x}=(0,0)$, we take a ray in a specific direction, starting at the radar position towards the outer boundary, using $r$ to indicate the distance from the radar. We write $s(r)$ for the elevation along the ray, and $h_{R}$ for the height of the radar. The straight line to the radar from a point $(r, s(r))$ at the sea surface at position $r$ is given for $\rho<r$ by $z=\ell(\rho, r)=s(r)+a(r-\rho)$ with $a=$ $\left(H_{r}-s(r)\right) / r$. The point $(r, s(r))$ at the sea surface is visible if $\ell(\rho, r)>s(\rho)$ for all $\rho<r$, i.e. if $\min _{\rho}(\ell(\rho, r)-s(\rho))>0$. At the boundary of such intervals the value is zero, and so the visible and invisible intervals are characterized by $\operatorname{sign}\left[\min _{\rho}(\ell(\rho, r)-s(\rho))\right]=0$ and $=-1$ respectively. This leads to the definition of the characteristic visibility function as

$$
\chi(r)=1+\operatorname{sign}\left[\min _{\rho}\{\Theta(r-\rho) \Theta(\rho)(\ell(\rho, r)-s(\rho))\}\right]
$$

where $\Theta$ is the Heaviside function, equal to one for positive arguments and zero for negative arguments. The visibility function equals 0 and 1 in invisible and visible intervals respectively. The shadowed wave ray, as seen by the radar, is 
then given by

$$
s_{\text {shad }}(r)=s(r) \cdot \chi(r)
$$

which defines the spatial shadow operator along the chosen ray. Repeating this process on rays through the radar for each direction, leads to the shadowed sea, $S_{\text {shad }}(\mathbf{x})$

The geometric image is obtained by removing information in a circular area around the radar position with a radius of $r_{0}$. Then the geometric image is described by

$$
I(\mathbf{x})=S_{\text {shad }}(\mathbf{x}) \cdot \Theta\left(|\mathbf{x}|-r_{0}\right)
$$

\section{Dynamic averaging-evolution scenario}

\section{This section presents the dynamic averaging-evolution scenario (DAES) that} will provide a reconstruction and prediction of the surface elevation at the radar position using the geometrically shadowed waves in the ring-shaped observation area of the sea. The main ideas can be described as follows.

The exact (non-shadowed) sea is supposed to evolve according to a linear (dispersive) evolution operator. Except from entrance effects of waves through the boundary, one snapshot of the sea would be enough to determine exactly the whole future evolution. The effects of shadowing give a space and time dependent perturbation for all images: the amount of shadowing (visibility) depends on the distance from the radar, and the position in time of the waves determines the actual area of shadowing, shifting and changing somewhat with the progression of the wave. Hence, one snapshot of the observed (shadowed) sea, will produce a different evolution result than that of the exact sea because the zero-level of the shadowed area will be evolved. In order to control, and actually reduce, the error, we use updates to be assimilated in the dispersive evolution. After three radar rotation times $3 d t$ we update the ongoing simulation by assim- 
ilation with the averaged 3 preceding images, where the averaging itself already reduces the effect of shadowing somewhat. Since we do this globally, so also in areas closer to the radar where the shadowing is less severe, the result with the dynamic averaging-evolution scenario shows that this is sufficiently successful to give an acceptable correlation in the radar area.

The first subsection deals with two simple methods that aim to improve the quality of each individual geometric image by attempting to fill in the gaps caused by the shadowing. Then the evolution of a single image is discussed in some detail, after which the dynamic averaging of several images is described to construct updates that will be used in subsection four as assimilation data in an evolution of the full sea.

\subsection{Spatial reconstruction of geometric images}

In the following, two methods will be presented for a first attempt to reconstruct the geometric images in regions where the observation is shadowed.

In the first method the geometric image is shifted vertically such that the spatial average (over the observation area) vanishes. With a scaling factor $\alpha$ to obtain the correct significant wave height, this will produce the reconstructions $R_{n}^{1}$ as

$$
R_{n}^{1}(\mathbf{x})=\alpha\left(I_{n}(\mathbf{x})-\operatorname{mean}\left(I_{n}\right)\right)
$$

As mentioned in the introduction, it is assumed that the true variance of the waves (or significant wave height) is known from either additional analysis and/or a reference measurement so that $\alpha$ is determined.

The second proposed method is described as

$$
R_{n}^{2}(\mathbf{x})=\alpha\left(I_{n}(\mathbf{x})-E\left(I_{n},-T / 2\right)\right)
$$


Here $E\left(I_{n},-T / 2\right)$ evolves the sea backwards in time over half of the peak period, for which in multi-modal seas we will take the peak period of the wind waves. The evolution indicated here with the operator $E$ will be explained in detail in the next subsection. Note that for harmonic long crested waves with period $T$ of which negative elevations have been put to zero elevation (to roughly resemble the effect of shadowing) leads to the correct harmonic wave by the reconstruction $R^{2}$.

\subsection{Evolution of a single image}

Let the reconstructed geometric image, denoted by $R$, obtained by either reconstruction method described in the previous subsection, be given by its $2 \mathrm{D}$ Fourier description as:

$$
R(\mathbf{x})=\sum_{\mathbf{k}} a(\mathbf{k}) e^{i \mathbf{k} \cdot \mathbf{x}}
$$

Here $\mathbf{k}$ is the $2 \mathrm{D}$ wave vector, and the coefficients $a$ can be obtained by applying a 2 D FFT on $R$.

The image itself is not enough to define the evolution uniquely since the information in which direction the components progress with increasing time is missing. For given direction vector e, define the forward evolution as

$$
E_{\mathbf{e}}(R, t)=\sum_{\mathbf{k}} a(\mathbf{k}) \exp i[\mathbf{k} \cdot \mathbf{x}-\operatorname{sign}(\mathbf{k} \cdot \mathbf{e}) \Omega(k) t]
$$

where $k=|\mathbf{k}|$ and $\Omega(k)=\sqrt{g k \tanh (k D)}$ is the exact dispersion above depth $D$. Waves propagating in a direction $\tilde{\mathbf{e}}$ that makes a positive angle with $\mathbf{e}$, so $\tilde{\mathbf{e}} \cdot \mathbf{e}>0$, will then propagate in the correct direction for increasing time, which justifies to call the evolution forward propagating with respect to e. Changing the minus-sign into a plus-sign in the phase factor, the backward propagating evolution in the direction $-\mathbf{e}$ is obtained.

For uni-modal sea states, such as wind waves or swell, there will be a main 
propagation direction $\mathbf{e}_{\text {prop }}$, which is the direction of propagation of the most energetic waves. Other waves in such wave fields will usually propagate in nearby directions, under an angle less than $\pi / 2$ different from the main direction. In such cases we can take $\mathbf{e}_{\text {prop }}$ as the direction to define the evolution. Actually, any direction from the dual cone of wave vectors can be chosen, i.e. any vector that has positive inner product with all wave directions.

In multi-modal sea states, in most practical cases a combination of wind waves and swell, the situation is different since the waves may have a wider spreading than the $\pi / 2$ difference from the main direction that was assumed for the unimodal sea states. When the wave directions are spread out over more than a half space, one evolution direction so that all waves are propagated correctly cannot be found anymore. If only low-energy waves are outside a half space, one may still use a forward propagating evolution operator. Then an optimal choice is the main evolution direction for which the maximum portion of the total wave energy is evolved correctly. A way to identify this optimal direction is discussed now.

Practically, we use a second (or more) 'control' image, and look for which vector e the evolution of the first image corresponds with the control image as good as possible in least-square norm; this then determines the main evolution direction (MED). Explicitly, given two successive images of the wave field, say $R_{1}$ and $R_{2}$ a small time (the radar rotation time) $d t$ apart, we compare $R_{2}$ with the forward evolution of $R_{1}$ over time $d t$ in the direction $\mathbf{e}$, to be denoted by $E_{\mathbf{e}}\left(R_{1}\right)$, and minimize the difference over all directions e, defining the MED as the optimal value

$$
\mathbf{e}_{M E D} \in \min _{\mathbf{e}}\left|E_{\mathbf{e}}\left(R_{1}\right)-R_{2}\right|
$$

Instead of minimizing a norm of the difference, one can also take the maximum of the correlation. For fields with limited directional spreading there will be a 
broad interval of optimal directions, in which case the average of the optimal directions can be chosen. For cases of multi-modal sea states where the main propagation direction of the different modes deviate very much there is likely to be one distinct optimal MED. It is possible that with this method using the MED, a significant amount of wave energy is evolved in the wrong direction, depending on how much the main directions of the different modes differ.

In the following we will use a simplified notation when evolving over one time step $d t$, namely

$$
\mathcal{E}(R)=E_{\mathbf{e}_{M E D}}(R, d t)
$$

Evolving over several time steps, say m.dt, is then written as a power (succession of evolution) $\mathcal{E}^{m}$.

\subsection{Updates from dynamic averaging}

The reconstruction process described in subsection 3.1 gives approximate sea states $R_{n}$. The study cases will show that these reconstructions are still rather poor when compared to the exact synthetic surface elevation maps; the correlation with the exact surface is only slightly better than that for the shadowed geometric images. In order to reduce the effect of this reconstruction error and thereby to improve the accuracy of the elevation prediction near the radar, we propose an averaging procedure in physical space. This procedure will involve three successive reconstructed images and the simulated wave field at the instant of the last image.

To set notation, the simulated sea (the simulation process will be detailed below) at time $t$ will be denoted as $\zeta(\mathbf{x}, t)$; at discrete times $m$.dt we write $\zeta_{m}(\mathbf{x})=\zeta(\mathbf{x}, m \cdot d t)$

The simulation is initialized by taking for the first three time steps the three 
successive reconstructed images

$$
\zeta_{m}(\mathbf{x})=R_{m}(\mathbf{x}) \text { for } m=1,2,3
$$

For the continuation, updates will be used to assimilate the evolution. We describe the update process at a certain time $t_{0}$, which is a multiple of $3 d t$. Available at that time are the simulated wave field at $t_{0}$, to be denoted by $\zeta_{0}(\mathbf{x})=\zeta\left(\mathbf{x}, t_{0}\right)$, the reconstructed image at time $t_{0}$, and 2 previous images at times $t_{-1}=t_{0}-d t, t_{-2}=t_{0}-2 d t$; these reconstructed images will be denoted by $R_{0,-1,-2}$ respectively. Since the images $R_{k}$ have substantial inaccuracies despite the reconstruction, it can be expected that an averaging procedure improves the quality. This averaging has to be done in a dynamic way to compensate for the fact that the images are available at different instants in time. Therefore the images $R_{-1}$ and $R_{-2}$ have to be evolved over one, respectively two, time steps $d t$. This produces $\mathcal{E}\left(R_{-1}\right)$ and $\mathcal{E}^{2}\left(R_{-2}\right)$, each representing, just as $R_{0}$, an approximation of the sea state at time $t_{0}$. But the information will be different, partly complementary, because the information at different time steps shows somewhat different parts of the wave because of the shadowing effect. Therefore an arithmetic mean will contain more information, and may also reduce incidental errors and noise. The ongoing simulation $\zeta_{0}$ also gives an approximation of the sea at $t_{0}$, and, most important, will also contain elevation information in the near-radar area where the $R_{k}$ are vanishing. Choosing some weight factors, we therefore take as update at time $t_{0}$ the following combination

$$
U_{0}(\mathbf{x})=\left(\frac{1}{6}\left(R_{0}+\mathcal{E}\left(R_{-1}\right)+\mathcal{E}^{2}\left(R_{-2}\right)\right)+\frac{1}{2} \zeta_{0}\right)\left(1-\chi_{\text {rad }}\right)+\zeta_{0} \chi_{\text {rad }}
$$

Here $\chi_{\text {rad }}(\mathbf{x})$ is the characteristic function (or a smoothed version) of the nearradar area: $\chi_{\text {rad }}=1$ in the near radar zone where no waves can be observed 
and $\chi_{\text {rad }}=0$ in the remaining area. The number of reconstructed images to be taken in the update can be more or less than 3 , and each could be given a different weight. Our experience with various test cases led to the weight factors as taken above.

\subsection{Evolution and prediction}

The updates defined above will be used as assimilation data to continue the simulation. In detail, after the construction of an update, say $U_{3 m}$, the simulation continues with this sea state as initial elevation field for three consecutive time steps:

$$
\zeta_{3 m+j}=\mathcal{E}^{j}\left(U_{3 m}\right) \text { for } j=1,2,3 .
$$

This defines the full evolution in time steps $d t$, which is repeatedly fed with new information from the reconstructed images through the updates. This scenario can run in real time in pace with incoming real radar images.

A prediction can be defined, starting at any time $t_{0}=m . d t$ for a certain time interval ahead, without using any information of geometric images later than $t_{0}$. The prediction, say for a future time of $\tau \in[0, \Pi]$, where $\Pi$ is the prediction horizon, is then defined as

$$
P\left(t_{0}, \tau\right)=E\left(\zeta\left(t_{0}\right), \tau\right) \text { for } \tau \in[0, \Pi] \text {. }
$$

An upper bound for the prediction horizon depends on the speed of the waves and the distance of the outer boundary to the radar. As shown by $\mathrm{Wu}(2004)$ and Naaijen et al. (2014) the prediction horizon is mainly governed by the group velocity of the waves and the size of the observation domain. In case of a nautical radar, the spatial observation domain will be the ring-shaped area, previously indicated by $\chi_{\text {rad }}=0$. The group velocity will be different and in different directions for short-crested, in particular multi-modal, seas and depend 
on wave characteristics (roughly the peak period) and the depth. These factors will influence the prediction horizon in which we can expect a reliable prediction. Besides that, the prediction horizon $\Pi$ clearly also depends on the accuracy that is desired for the prediction.

\section{Case studies}

In this section we present the results for two study cases: one for wind waves and one with combined wind waves and swell. Comparisons are presented between the predicted wave elevation, obtained by processing the synthesized images with the proposed DAES method and the exact wave elevation as it was synthesized. We start to specify the sea data and other physically and numerically relevant parameters of the simulations, followed by the simulation results.

\subsection{Parameters of the study cases}

\subsubsection{Geometry and spatial grid parameters}

The seas that we consider evolve above a depth $h=50 \mathrm{~m}$. The height of the radar is an important quantity because the severity of the shadowing effect is governed by the ratio of radar height and wave height. We will report on a value of the radar height $h_{R}$ of $15 \mathrm{~m}$ above the still water level. The radar is assumed to be at a fixed position above the still water level, with a constant radar rotation speed $d t=2 \mathrm{~s}$. The sea is constructed in an area $[-2050,2050]^{2}$ with a number of nodes in $x$ and $y$-direction equal to $N_{x}=N_{y}=512$, so spatial step size $d x=d y=7.9 \mathrm{~m}$. Modeling the outer boundary of the radar observation area, the elevation of each snapshot of the sea is made to vanish for distances from the radar larger than $r_{\max }=1800 \mathrm{~m}$. The shadowing procedure is applied after transforming each sea state to polar coordinates $(r, \phi)$ on a grid with $d r=7.5 \mathrm{~m}$ and $d \phi=0.3^{\circ}$. The geometric image is then obtained by 

$h=50 \mathrm{~m}$, are summarized in Table 1.

Table 1: Characteristic of sea and swell waves

\begin{tabular}{|c|c|c|c|c|c|c|c|c|c|c|}
\hline Sea & $H_{s}$ & $T_{p}$ & $\gamma$ & $\theta_{\text {main }}$ & $s$ & $\omega_{p}$ & $k_{p}$ & $\lambda_{p}$ & $C_{p}$ & $V_{g}$ \\
\hline Wind & 3 & 9 & 3 & $-\pi / 2$ & 10 & 0.7 & 0.05 & 125 & 13.9 & 7.4 \\
\hline Swell & 1 & 16 & 9 & $3 \pi / 4$ & 50 & 0.4 & 0.02 & 308 & 19.2 & 14.8 \\
\hline
\end{tabular}

371

transforming back to Cartesian coordinates and make the elevation vanish in the circular near radar area of radius $r_{\min }=500 \mathrm{~m}$.

\subsubsection{Sea states}

We provide the properties of the wind waves and the swell separately; since we consider linear waves, the characteristics of the multi-modal sea state, which is a combination of the wind waves and swell, can be derived in a straightforward way. The properties of the waves, with related wave characteristics above depth

The wind waves have main propagation direction from North to South, $\theta^{W}=$ $-\pi / 2$; the wave spreading is given by the spreading function (1) with exponent $s=10$.

The frequency spectrum of the wind waves is a Jonswap spectrum with $\gamma=3$, peak period $T_{p}=9 \mathrm{~s}$, and significant wave height $H_{s}^{W}=3 \mathrm{~m}$. Note that the significant wave height is an important factor that affects the amount of shadowing; the ratio of radar height and significant wave height is as low as 5 in this study case, leading to substantial shadowing.

The multi-modal sea consists of the above wind waves to which is added the swell waves. The swell consists of waves from the South-Eastern direction, $\theta^{S}=3 \pi / 4$, peak enhancement factor $\gamma=9$, wave spreading with $s=50$, peak period $T_{p}=16 \mathrm{~s}$, and significant wave height $H_{s}^{S}=1 \mathrm{~m}$. The significant wave height of this combined sea state will be $H_{s}^{W S}=\sqrt{10} \approx 3.15 \mathrm{~m}$, so that the ratio of radar height and significant wave height is slightly less than 5 .

The study cases of wind waves without swell and combined wind waves-swell will 
be denoted by W15 and WS15 respectively. The number of discrete components $N$ used to synthesize the waves as in equation (2), has been taken $N=1500$ for the wind waves and $N=700$ for the swell in study case WS15.

\subsubsection{Main evolution direction}

As described in subsection 3.2, the main evolution direction MED will be determined as the direction for which the error of the difference between a onestep evolved image and the successive image is as small as possible. For the study cases Figure 1 shows the averaged relative error obtained by comparing 10 pairs of successive reconstruction images for case W15 and WS15. Here, the angle is measured from the positive $x$-axis in counter clockwise direction. For study case W15 the relative error is rather constant in the interval $\left[-150^{\circ},-30^{\circ}\right]$, with $-90^{\circ}$ in the middle of the interval. Hence this is chosen as MED, which coincides with the design value of the main wind direction of the synthesized wave field. For case WS15 the situation is very different. There is now only a small interval of angles identifying evolution directions for which most energy is propagated correctly. Hence, for case WS15 the angle of minimal error is chosen as MED, i.e. $-148^{\circ}$. For the study cases using the shadowed images to determine MED we observed a few degrees difference with the MED's found when using the synthetic non-shadowed seas; in the following we take the values obtained from the shadowed seas.

\subsection{Simulation Results}

In this paragraph results of the simulations will be described. After some graphical presentations, more quantitative information is presented for the reconstruction sea states and the future prediction. 


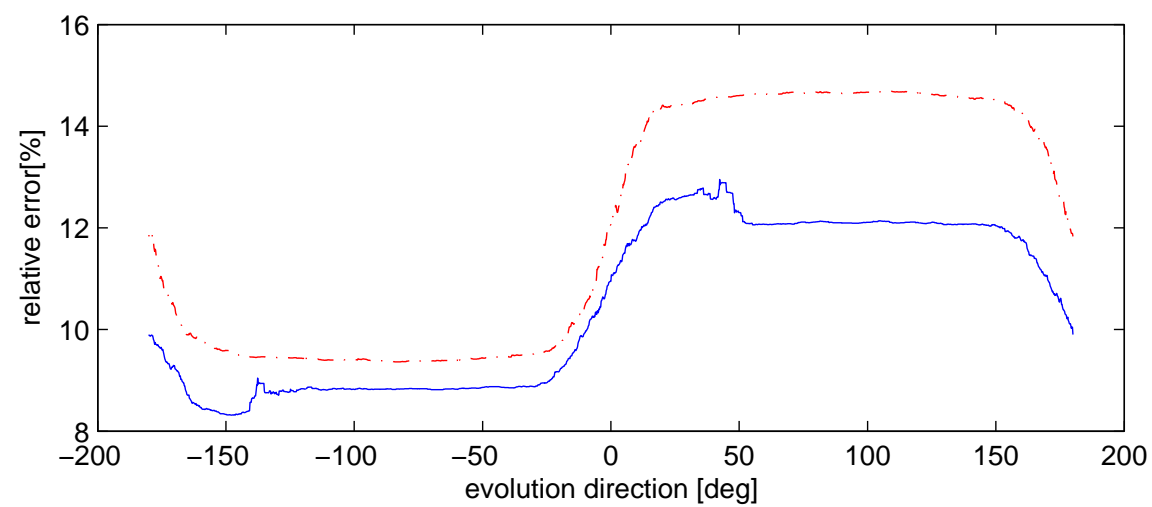

Figure 1: The relative error in the procedure to determine the main evolution direction MED averaged over 10 realizations, for case W15 (dash-dotted red) and WS15 (solid blue).

\subsubsection{Graphical presentation}

We start with some results that illustrate the DAES method. After the first three synthesized geometric images, the dynamic averaging - evolution scenario is initiated using updates at every time that is a multiple of $3 d t$. For a certain $t=t_{0}$, shortly after starting the simulation, various images are presented in Figure 2. Figure 2a shows the true wave elevation as synthesized at $t=t_{0}$. Figure $2 \mathrm{~b}$ shows the shadowed image of the wave elevation depicted in Figure 2a with vanishing elevation in the blind area $r<500$ around the antenna. Figure 2c, shows the reconstruction $U_{0}\left(t_{0}\right)$ (also denoted by $P\left(t_{0} ; \tau=0\right)$ ). As can be seen, the wind waves propagating in the main direction from North to South in the negative y-direction, and more so the swell from SE to NW, have evolved already some small distance into the near-antenna zone. Figure 2d shows the reconstruction $P\left(t_{1} ; \tau=0\right)$ for a larger value $t_{1}$ at which the waves have evolved so much that they occupy the entire blind area near the antenna $r<500$.

Figure 3 shows the cross section in the $y$ direction at $x=0$ of the shadowed waves in Figure 2b. Different from Figure 2b, the waves are shown here for $r<r_{\min }$ as well. As can be observed for this particular wave condition and quotient of radar altitude and significant wave height of $15 / 3$, the shadowing 


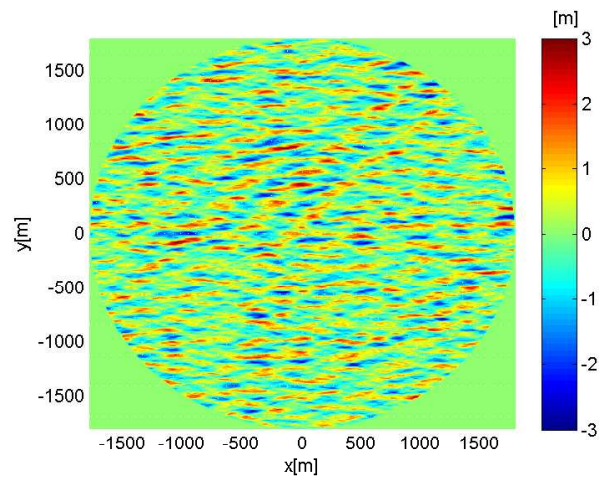

(a)

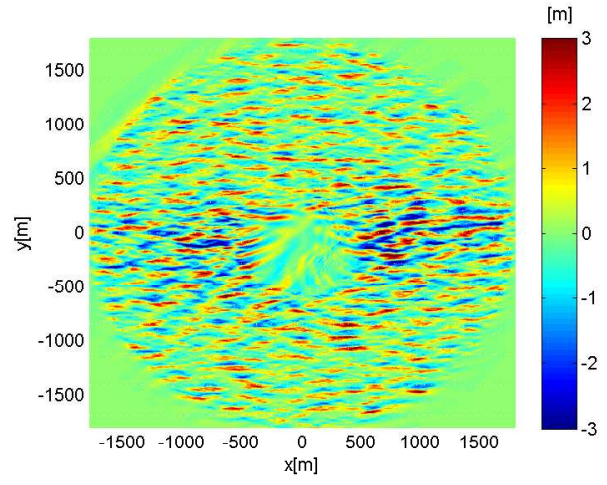

(c)

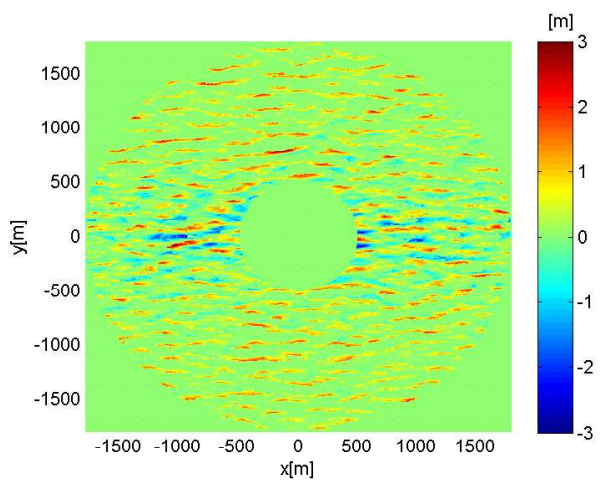

(b)

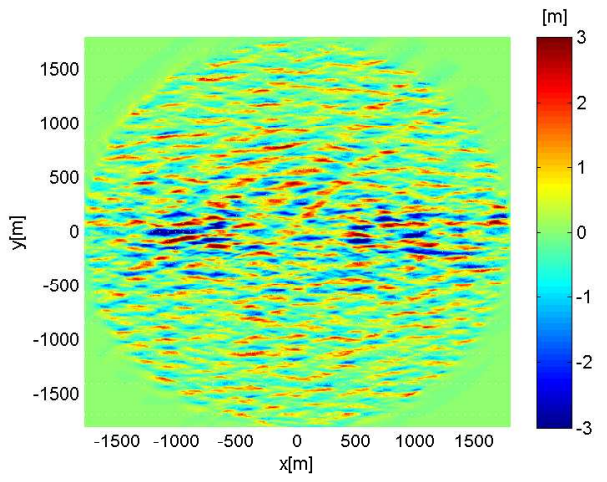

(d)

Figure 2: Images of the combined sea WS15 with wind waves from the North and swell from SE. Image (a) shows the real sea, and (b) the shadowed sea at the same instant. Image (c) shows the elevation shortly after the start of the simulation when the waves do not yet fully occupy the blind near radar area; at a later time, image (d) shows that the blind area has been filled with waves through the dynamic averaged evolution scenario. 


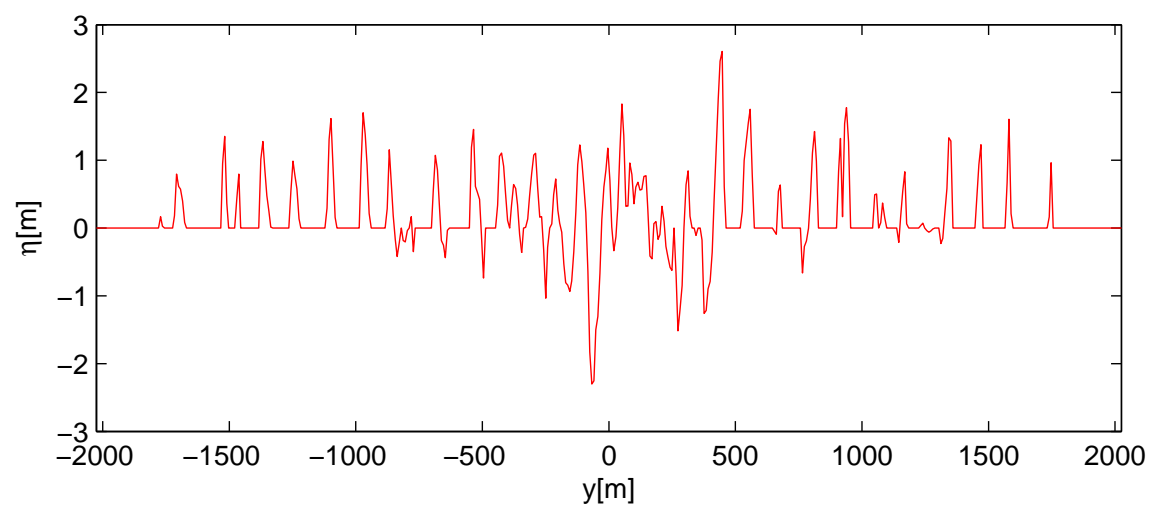

Figure 3: A cross section coinciding with the $y$-axis shows the shadowed waves (wind waves from right to left); observe the severe shadowing outside the blind area $(-500,500)$.

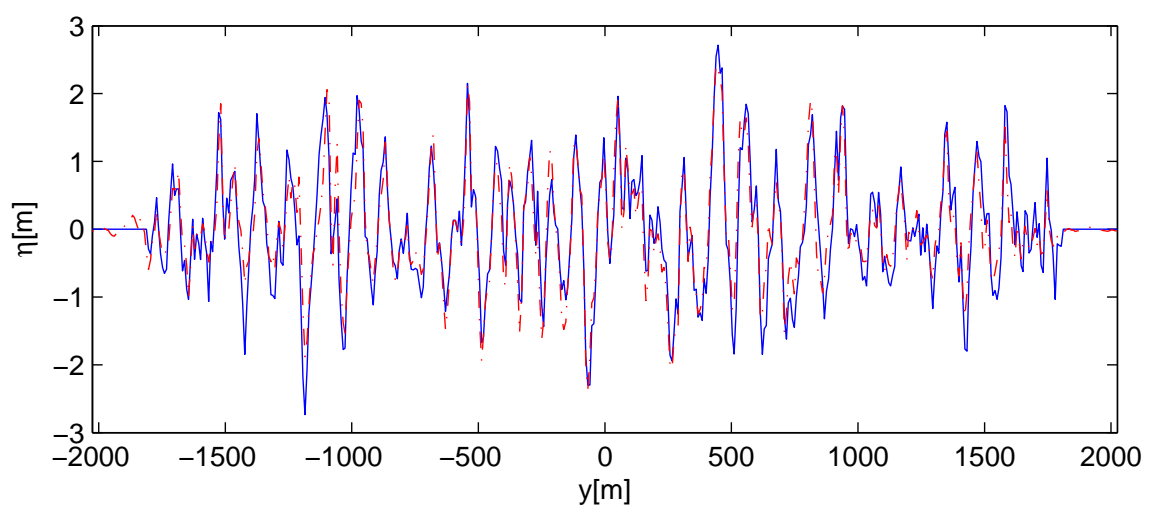

Figure 4: Cross section along $y$-axis showing the true elevation (blue,solid) and the reconstructed elevation $R^{1}$ (red, dashed).

is rather severe: beyond $r=500$ hardly any wave troughs are visible. Despite this poor quality of the observation, the DAES procedure produces a reconstruction of the wave elevation as shown in Figure 4. This figure shows plots of the synthesized elevation, referred to as "true wave", and the reconstruction $P\left(t_{1} ; \tau=0\right)$ obtained by DAES at a time $t_{1}$ such that the simulation has already run sufficiently long for the reconstructed waves to fill the entire blind zone. Observe that the reconstruction is better near the radar, near $y=0$, than for larger distances from the radar where the dynamic averaging cannot 

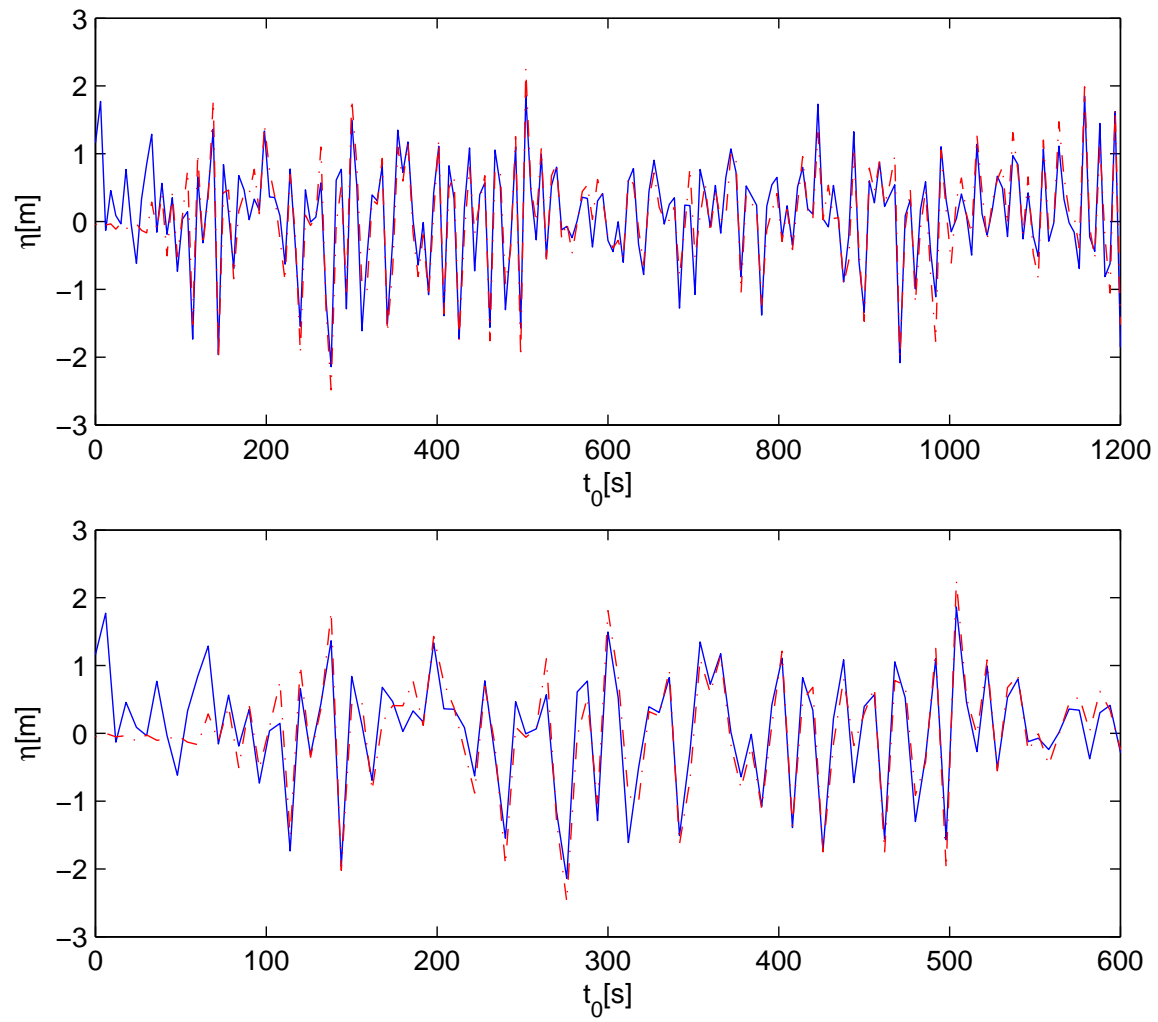

Figure 5: Time traces of elevation at the radar position for case WS15. In blue (solid) the true elevation, in red (dashed) the reconstruction $R^{1}$ that started at time 0 . The enlarged lower plot from 0 to $300 \mathrm{~s}$ shows the entrance effect that only after some $80 \mathrm{~s}$ the faster and slower waves reached the radar position to obtain sufficient accuracy. 


\subsubsection{Correlation as measure for accuracy}

The accuracy of the reconstruction and prediction is quantified by the correlation coefficient Corr, which correlates the wave elevation at one instant obtained from the simulation ('simul') with the synthetic wave elevation ('data') at the same instant according to

$$
\operatorname{Corr}(\text { data, simul })=\frac{<\text { data, simul }>}{\mid \text { data }|| \operatorname{simul} \mid}
$$

451 Here $<,>$ denotes the inner product over space $\mathbf{x}$. Note that Corr defined in this way is related to the normalized point square error according to

$$
\frac{\mid \text { data }- \text { simul }\left.\right|^{2}}{\mid \text { data }|\cdot| \text { simul } \mid}=\frac{\mid \text { data } \mid}{\mid \text { simul } \mid}+\frac{\mid \text { simul } \mid}{\mid \text { data } \mid}-2 \operatorname{Corr}(\text { data, simul })
$$

In particular when 'data' and 'simul' have the same norm, it holds

$$
\frac{|d a t a-\operatorname{simul}|^{2}}{\mid \text { data }\left.\right|^{2}}=2(1-\operatorname{Corr}(\text { data }, \text { simul }))
$$

454 The correlation will also be used to quantify the quality of future predictions.

455 Using the notation $P\left(t_{0}, \tau\right)$ introduced in equation (14) for the predicted wave elevation starting with the reconstruction at time $t_{0}$ a time $\tau$ ahead, and denoting by $\eta\left(t_{0}+\tau\right)$ the synthetic wave elevation from equation (2), their spatial correlation will be denoted by

$$
c\left(t_{0}, \tau\right)=\operatorname{Corr}\left(P\left(t_{0}, \tau\right), \eta\left(t_{0}+\tau\right)\right) .
$$


Then in order to obtain a statistically more reliable average correlation coefficient corr, the average is taken over an interval of $t_{0}$ values:

$$
\operatorname{corr}(\tau)=\frac{1}{J} \sum_{j=1}^{J} c\left(t_{0 j}, \tau\right)
$$

To avoid entrance effects, the computation of $\operatorname{corr}(\tau)$ is restricted to times $t_{0}$ such that all waves have evolved to fill completely the blind zone. For the presented simulations, this distance (of $1000 \mathrm{~m}$ ) is covered by the wind waves with group speed at peak frequency in approximately 136 s, i.e. $68 d t$; for the swell waves with double group speed, this time is $68 \mathrm{~s}$. The number of simulation steps $J$ used for calculation of $\operatorname{corr}(\tau)$ has been at least 200 for all presented results.

\subsubsection{Accuracy of reconstruction}

The correlation has been computed for both sea states W15 and WS15, for various sizes of the spatial domain: corr is determined for $r<50, r<500$ and $r>500$. Results are presented in Tables 2 and 3 for the 'reconstruction', i.e. $\tau=0$; prediction results for which $\tau>0$ will be presented in the next paragraph.

The first column in Tables 2 and 3 indicates the type of input data used in the DAES procedure. 'Sea' refers to the perfect (not shadowed) synthetic waves as input images, but with vanishing elevation in the near radar area $r<500$.

In this column $R^{0}$ refers to simulations with shadowed waves without applying any reconstruction of the individual images, while $R^{1}$ and $R^{2}$ refer to the two reconstruction methods as defined in subsection 3.1 .

The columns with 'Raw' and 'Rec' show the correlation of the geometric images and the individually reconstructed images with the true wave elevation respectively; the area over which the correlation is taken is the outer ring area 
$500<r<1800$.

Table 2: Correlation for W15 averaged over time for various reconstruction methods.

\begin{tabular}{cccccc}
\hline & Raw & Rec & $r<50$ & $r<500$ & $r>500$ \\
\hline Sea & 1.00 & 1.00 & 0.99 & 0.99 & 1.00 \\
$R^{0}$ & 0.71 & 0.71 & 0.82 & 0.87 & 0.83 \\
$R^{1}$ & 0.71 & 0.75 & 0.95 & 0.95 & 0.89 \\
$R^{2}$ & 0.71 & 0.75 & 0.89 & 0.91 & 0.84 \\
\hline
\end{tabular}

Table 3: Same as 2 now for bi-modal sea state WS15.

\begin{tabular}{cccccc}
\hline & Raw & Rec & $r<50$ & $r<500$ & $r>500$ \\
\hline Sea & 1.00 & 1.00 & 0.99 & 0.99 & 1.00 \\
$R^{0}$ & 0.70 & 0.70 & 0.85 & 0.88 & 0.83 \\
$R^{1}$ & 0.70 & 0.74 & 0.95 & 0.95 & 0.89 \\
$R^{2}$ & 0.70 & 0.73 & 0.89 & 0.90 & 0.83 \\
\hline
\end{tabular}

As illustration, for a typical case, the correlation between the true sea and the $R^{1}$-reconstruction in the radar area $(r<50 \mathrm{~m}$ and $r<200 \mathrm{~m})$ is given in Figure 6 as function of increasing time during the DAES process. The entrance effect is clearly visible just as in Figure 5; the waves need approximately $160 \mathrm{~s}$ to fill up the near-radar area of radius $200 \mathrm{~m}$.

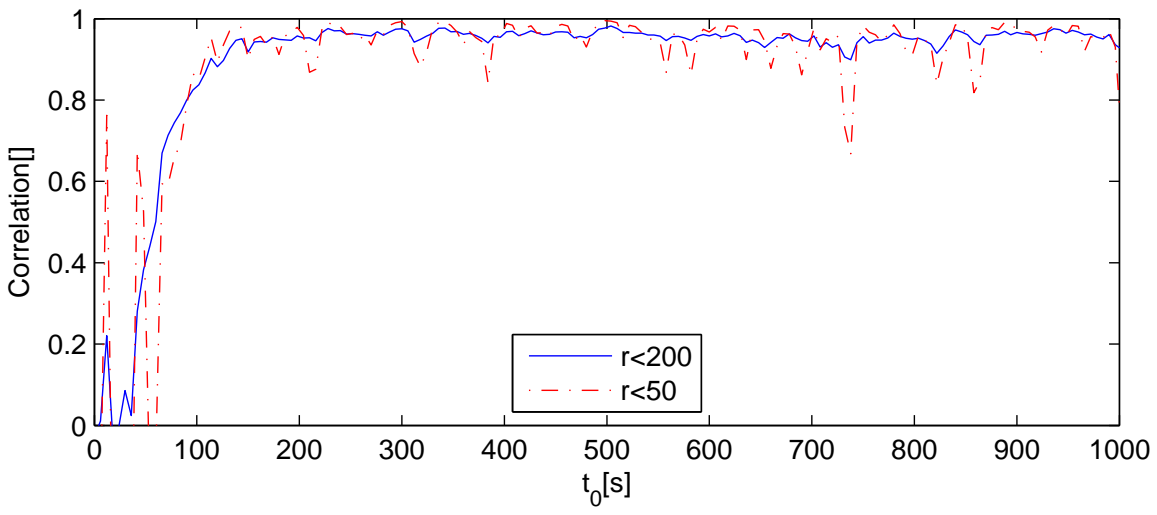

Figure 6: Correlation between true sea and the $R^{1}$-reconstruction for case WS15 in the radar area with radius $200 \mathrm{~m}$ (blue) and radius $50 \mathrm{~m}$ (red) at times after the start of the reconstruction. Observe that after some $160 \mathrm{~s}$ the reconstruction has filled these regions and becomes more accurate. 

elevation at the radar position are shown as function of prediction time $\tau$.

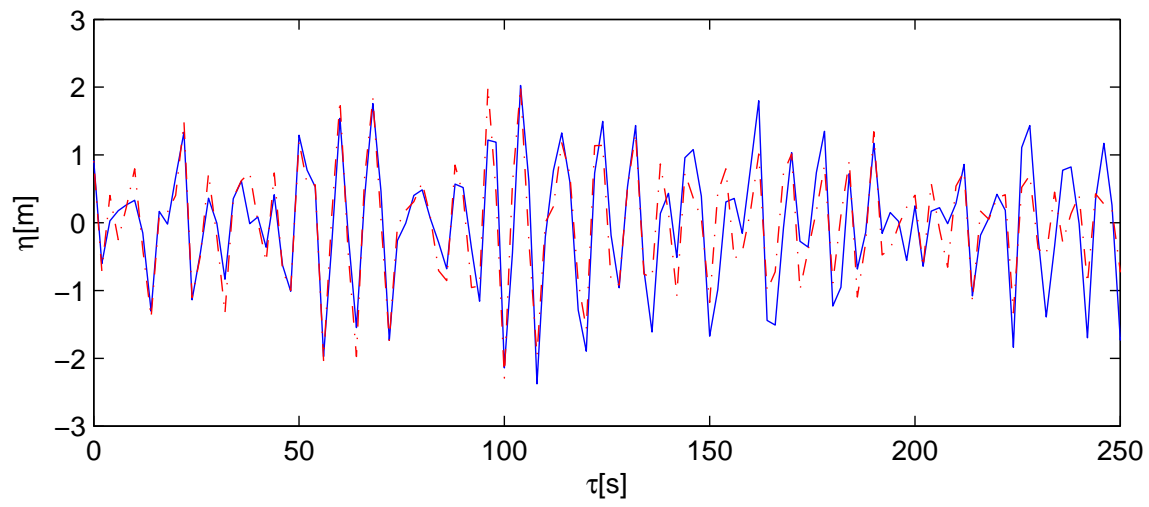

\subsubsection{Accuracy of prediction}

The eventual aim of the simulation scenario is to predict in future time the elevation in the near-radar area. At each time $t_{0}$ during the simulation, the obtained reconstruction at that time $P\left(t_{0}, \tau=0\right)$ can be taken as initial state for a prediction according to equation (14), without new updates. In Figure 7 is shown a prediction at the radar position for the sea state WS15 with reconstruction method $R^{1}$. For an initial time $t_{0}>160$ larger than the filling time of the near-radar area, the predicted wave elevation and the true wave

Figure 7: For WS15, the figure shows the prediction (red,dashed) of the elevation compared to the true elevation at the radar position; observe that after $120 \mathrm{~s}$ the prediction becomes less accurate.

Figures 8 and 9 show results for prediction based on DAES applied to the true sea (perfect non-shadowed waves) and the $R^{1}$-reconstruction for case W15 and WS15 respectively. As expected, for increasing prediction time the correlation decreases. Prediction of the wind waves W15 can be done for a time horizon of 2.9 minutes with correlation above 0.9 , and for 3.6 minutes with correlation above 0.8; for the combined wind-swell waves WS15 these times are 2 minutes and 3.3 minutes respectively. Observe the steeper decrease in the graphs of WS15 after $120 \mathrm{~s}$, which is approximately the travel time of swell waves at 

prediction anymore.

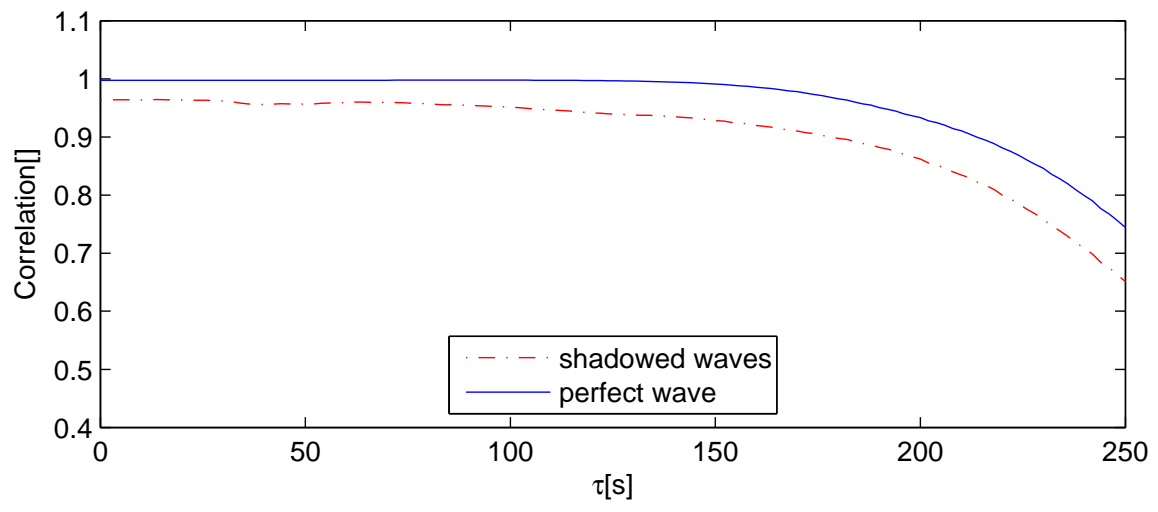

Figure 8: Correlation between predicted and true elevations in a radar area of radius $200 \mathrm{~m}$ using as input in the prediction method the true sea (blue, solid) and the shadowed sea of W15 (red, dashed).

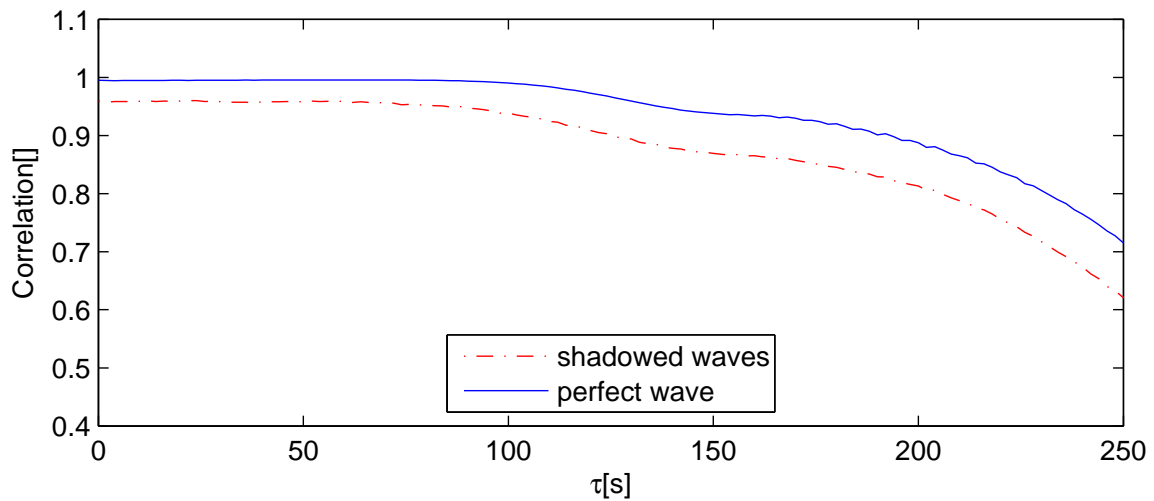

Figure 9: Same as Figure 8 now for WS15.

\section{Discussion of results}

\subsection{Reconstruction method}

The high correlations in Tables 2 and 3 for the case of a perfect 'Sea' (the nonshadowed synthetic waves) as input, show that the dynamic averaging procedure 
and the evolution to fill the near-radar area $r<500$ proceeds almost perfectly. The tables also show that the reconstruction of each single image only slightly improves the correlation, at most $4 \%$ for $R^{1}$ and $R^{2}$. For all three individual reconstructions, the DAES improves the reconstruction substantially, with best results for the vertical shifting method $R^{1}$, for which the correlation increases from 0.75 in the outer ring to 0.95 in the near-radar area.

The comparison of the $R^{1}$-reconstructed and true elevation in Figure 6 shows that variations of the correlation over the larger disc of radius $200 \mathrm{~m}$ are much smaller than over the $50 \mathrm{~m}$ disc; this may be due to a poor reconstruction of relatively small areas in the outer ring $500<r<1800$.

\subsection{Predictability}

The results in Figures 8 and 9 show the capabilities and limitations of the prediction. The physically maximal prediction time can be roughly estimated as the travel time from the outer region towards the radar $(1800 \mathrm{~m})$ for the most energetic waves at peak frequency. Using the value of the group velocity of the wind waves of $7.4 \mathrm{~m} / \mathrm{s}$, this leads to a maximal prediction horizon of $240 \mathrm{~s}$ for study case W15; this seems to be a too high estimation since Figure 8 shows a rather low correlation of 0.7 at that time for the best possible prediction with the true sea.

On the other hand, for the combined wind-swell sea, a similar reasoning based on the speed of swell waves is too pessimistic for the study case WS15: the correlation of prediction with the true sea is around 0.9 at that time. This can be explained by the fact that in the study case the swell waves have approximately $10 \%$ of the energy of the wind waves, which causes that the wind waves dominate the correlation, which is only slightly less than for W15 until $250 \mathrm{~s}$, despite the fact that the effects of swell are actually absent after $120 \mathrm{~s}$. The swell effect can also be observed by comparing the predicted elevation with the true elevation 
at the radar position as depicted in Figure 7; the amplitude prediction becomes less accurate after around $120 \mathrm{~s}$ although the phase is still captured quite well for longer times.

\subsection{Scaling}

The observation from Figure 7 that the variance of the predicted wave elevation decreases with increasing $\tau$ is also due to the fact that for values of $\tau$ further into the future, the waves arriving at the radar location originate from further distances where the shadowing is more severe and the variance of the observation is lower; after sufficiently long time no wave information will be available at all anymore. Using one scaling factor $\alpha$ based on the variance of the entire observed image and the true variance of the waves as was proposed in equation (6), does not take into account this decreased visibility at large ranges from the radar and in fact does not even guarantee a correct variance at the radar for $\tau=0$. An alternative which is supposed to be practical and feasible for real life applications is proposed by Naaijen and Wijaya (2014): a time history of the wave elevation at the radar position (e.g. by an auxiliary wave buoy or via recorded ship motions) and a time history of the predicted wave elevation can be recorded and used to calculate the variance of the true waves and the prediction. By taking the ratio of these variances, a scaling factor dedicated for the radar location can be obtained. Such a scaling factor can also be computed as a function of $\tau$, thus removing the aforementioned effect of decreasing variance of the prediction with increasing $\tau$.

\subsection{MED and bimodal sea state}

In subsection 3.2 it was explained how the wave components obtained from a 2D FFT are propagated in the main evolution direction (MED). In case of multi-modal sea states, it depends on the difference between the propagation 
directions of the various modes how much of the total wave energy represented by the obtained components is propagated in the correct direction. The sea state WS15 was designed in such a way that the amount of energy represented by wave components propagating in opposite directions relative to the total wave energy is very limited which may explain the small differences in the obtained accuracy between W15 and the multi-modal case WS15. Multi-modal seas with substantial counter propagating waves require an evolution method that takes into account a splitting of waves in two opposite directions. Information from the directional spectrum can be used for this splitting, see Atanassov et al. (1985).

\subsection{Parameter dependence and robustness}

It has been remarked already that the dimensionless quantity in the vertical direction that determines the effects of shadowing is the ratio of radar height and significant wave height: the larger this ratio, the less effect of shadowing at a fixed position. This has been confirmed for other study cases that will not be reported here. The dimensionless quantity in the horizontal direction is the ratio of distance to the radar and the peak wave length, and has the same consequence. The length of the maximal prediction interval in case of multimodal sea states will depend in a somewhat complicated way on the relative energy contents and the difference of group speed of the wind waves and swell. For the study case described above (with 3 times larger significant wave height for the wind and with 2 times faster speed of the swell) the correlation as measure of quality seems to be too crude to identify the full effect of the swell; yet in observations of the spatial plots (or on cross sections) the difference can be noticed somewhat.

As is already indicated in Tables 2 and 3, almost irrespective the reconstruction of the shadowed seas, the DAES process produces substantially improved results 
in the near-radar area, with correlations between 0.88 and 0.95 depending on the reconstruction method. This robustness of the dynamic averaging and evolution scenario was also observed in other simulations. As an example, one other study case considered much wider spreading in the wind waves and swell. Although given by the same parameters as reported here, the argument $\theta-\theta_{\text {main }}$ in the spreading function was divided by 2 (which is sometimes also used). As a consequence, there is more overlap between the two sea states, and hence more counter propagating waves that will be evolved in the wrong direction. Nevertheless, correlations above 0.9 were obtained in the near radar area. A possible explanation for this seemingly inconsistent observation is that the much shorter waves cause less shadowing which may be a compensation in the measure given by the correlation.

\section{Conclusions and remarks}

In this paper we introduced a relatively simple and efficient simulation scenario to transform sequences of synthetic X-band radar images of multi-modal sea states into future sea states. The scenario turned out to be rather robust and produces reconstruction of the surface elevation in the blind area with correlation above 0.90 for the case of wind and wind-swell seas, for a ratio of radar height and significant wave height of 5. Additional simulations show that the correlation improves somewhat for higher values of this ratio because the effect of shadowing becomes less. No substantial differences are obtained for seas consisting of uni-modal wind waves or for multi-modal wind-swell seas.

The actual computation time for the simulation with the assimilation can run in real time; the required Fourier transforms for the averaging and evolution are executed within fractions of real time. For nonlinear simulations this may be somewhat longer but will not jeopardize the possibility to run the dynamic 
averaging-evolution scenario in real time.

The dynamic averaging-evolution scenario providing updates for a running evolution can be used in other cases also when a dynamic system experiences perturbations. We close with mentioning some topics worth of further investigations and possible improvements.

The simplification to consider linear seas above constant depth in this paper is mainly for ease of presentation and execution of the scenario; nonlinear seas above topography could be dealt with straightforwardly. Apart from this, our understanding of waves in real seas still seems to be quite rudimentary. Even for linear waves, concepts as the main evolution direction introduced here have not yet been related to energy propagation direction; the MED for WS15 is remarkably different from the direction of the main energy carrying wind waves that determines the direction of the change of the wave profiles during evolution. Besides that, detailed studies of nonlinear seas may show phenomena that are not captured by linear seas, such as the occurrence and physical processes that lead to freak-like waves. If coherent interference is the main process for the appearance of long crested freak waves with relatively low Benjamin-Feir index, as indicated by Slunyaev et al. (2005), Gemmrich and Garrett (2008) and Latifah and van Groesen (2012), the same process may also lead to freak waves in short crested waves, enhanced by nonlinear interaction processes.

In the reconstruction process in this paper, we assumed the significant wave height of the sea to be given. Recent investigations showed that this information can actually also be extracted from the geometric images, see Wijaya and van Groesen (2015).

Practical applicability requires the application of the full simulation scenario to real radar images and to test the results against accurate measurements. Another item to be clarified is if the accuracy of the predicted sea in the inner- 
radar area as achieved here, is sufficiently high to obtain accurately the forces on the ship carrying the radar, a topic of direct relevance for various practical applications. Finally, perturbations from heavy wind bursts may influence the results; it would be interesting and relevant to investigate the effects.

\section{Acknowledgments}

We appreciate very much the discussions and suggestions during meetings with the members of the participating groups in the Industrial Research Project PROMISED, executed together with MARIN, OceanWaves GMBH, Allseas, Heerema Marine Contractors and IHC Merwede, with financial support of the Dutch Ministry of Economical Affairs, Agentschap NL.

Alpers, W.R., Ross, D.B., Rufenach, C.L., 1981. On the detectability of ocean surface waves by real and synthetic aperture radar. J. Geophys. Res. 86(C7), 6481-6498.

Aragh, S., Nwogu, O., 2008. Variation assimilating of synthetic radar data into a pseudo-spectral wave model. J. Coastal Research : Special Issue 52, 235-244.

Atanassov, V., Rosenthal, W., Ziemer, F., 1985. Removal of ambiguity of twodimensional power spectra obtained by processing ship radar images of ocean waves. J. Geophys. Res. 90(C1), 10611067.

Blondel, E., Naaijen, P., 2012. Reconstruction and prediction of short-crested seas based on the application of a 3D-FFT on synthetic waves : Part 2Prediction. In : Proceedings of the 31st International Conference on Ocean, Offshore and Arctic Engineering, Rio de Janeiro, Brazil, pp. 55-70.

Borge, J.C.N., Reichert, K., Dittmer, J., 1999. Use of nautical radar as a wave monitoring instrument. Coastal Engineering 37, 331-342. 
Borge, J.C.N., Rodriguez, G.R., Hessner, K., Gonzalez, P.I., 2004. Inversion of Marine Radar Images for Surface Wave Analysis. J. Atmos. Oceanic Technol. 21, 1291-1300.

Buckley, J.R., Aler, J., 1998. Enhancements in the determination of ocean surface wave height from grazing incidence microwave backscatter. In : Proceedings of IEEE IGARS, Seattle, USA, pp. 2487-2489.

Clauss, G.F., Kosleck, S., Testa, D., 2012. Critical situations of vessel operations in short crested seas forecast and decision support system. J. Offshore Mech. Arct. Eng. 134(3), 031601.

Dankert, H., Rosenthal, W., 2004. Ocean surface determination from X-band radar image sequence. J. Geophys. Res. 109, C04016.

Gangeskar, R., 2014. An algorithm for estimation of wave height from shadowing in X-band radar sea surface images. IEEE Trans. Geosci. Remote Sens. 52, 3373-3381.

Gemmrich, J., Garrett, C., 2008. Unexpected Waves. J. Phys. Oceanogr. 38, 23302336 .

Goda, Y., 2010. Random Seas and Design of Maritime Structures, 3rd edition, World Scientific, Singapore.

Jefferys, E., 1987. Directional seas should be ergodic. Appl. Ocean Res. 9, 186191.

Latifah, A.L., Van Groesen, E., 2012. Coherence and predictability of extreme events in irregular waves. Nonlin. Processes Geophys. 19, 199-213.

Naaijen, P., Blondel, E., 2012. Reconstruction and prediction of short-crested seas based on the application of a 3D-FFT on synthetic waves : Part 1- 
Reconstruction. In : Proceedings of the 31st International Conference on Ocean, Offshore and Arctic Engineering, Rio de Janeiro, Brazil, pp. 43-53.

Naaijen, P., Wijaya, A.P., 2014. Phase resolved wave prediction from synthetic radar images. In : Proceedings of 33rd International Conference on Ocean, Offshore and Arctic Engineering OMAE, ASME, San Fransisco, USA.

Naaijen, P., Trulsen, K., Blondel, E., to be published. Limits to the extent of the spatio-temporal domain for deterministic wave prediction.

Plant, W.J., Farquharson, G., 2012a. Wave shadowing and modulation of microwave backscatter from the ocean. J. Geophys. Res. 117, C08010.

Plant, W.J., Farquharson, G., 2012b. Origins of features in wave numberfrequency spectra of space-time images of the ocean. J. Geophys. Res. 117, C06015.

Slunyaev, A., Pelinovsky, E., Soares, C.G., 2005. Modeling freak waves from the North Sea. Appl. Ocean Res. 27, 12-22.

Wijaya, A.P., Van Groesen, E., to be published. Determination of the significant wave height from shadowing in synthetic radar images.

Wu, G., 2004. Direct Simulation and Deterministic Prediction of Large-scale Nonlinear Ocean Wave field. Ph.D. thesis. Massachusetts Institute of Technology.

Young, I.R., Rosenthal, W., Ziemer, F., 1985. A three dimensional analysis of marine radar images for the determination of ocean wave directionality and surface currents. J. Geophys. Res. 90, 1049-1059.

Ziemer, F., Rosenthal, W., 1987. On the Transfer Function of a Shipborne Radar for Imaging Ocean Waves. In : Proceedings IGARSS'87 Symp., Ann Arbor, pp. 1559-1564. 